\title{
QUASI-INVARIANCE OF THE YANG-MILLS EQUATIONS UNDER CONFORMAL TRANSFORMATIONS AND CONFORMAL VECTOR FIELDS
}

\author{
THOMAS P. BRANSON
}

\section{Introduction}

It is well-known that the Yang-Mills equations on Minkowski space admit as an invariance group the 15-parameter group of conformal, or Lorentz angle-preserving transformations. We consider here what happens in the case of a conformal transformation $h$ between two finite-dimensional oriented pseudoriemannian manifolds $M$ and $N$ of arbitrary dimension and signature.

The Yang-Mills equations give a nonlinear condition $y(A)=0$ on a Lie algebra-valued one-form over $M$ or $N$. Quasi-invariance relations give formulas for $y\left(h^{*} A\right)$, and thus measure the obstruction to $h^{*} A$ satisfying the equations. This obstruction vanishes when $\operatorname{dim} M=4$ or when $h$ actually multiplies the metric tensor by a constant. Similar results hold for quasiinvariance of the linearized equations under conformal transformations and under Lie derivation with respect to conformal vector fields.

\section{The Yang-Mills equations}

Let $M$ be a smooth $\left(C^{\infty}\right)$ oriented pseudoriemannian manifold, with metric tensor $g$ of signature $(k, q), k+q=m=\operatorname{dim} M$. The inner product $g_{x}$ on tangent spaces $M_{x}$ given by $g$ induces a nondegenerate inner product on cotangent spaces $M_{x}^{*}$ upon identification of $M_{x}$ with $M_{x}^{*}$ through $g_{x}$. This in turn induces a nondegenerate inner product (also called $g_{x}$ ) on the exterior products $\Lambda^{p}\left(M_{x}^{*}\right)$, which may be characterized by

(2.1) $g_{x}\left(\omega^{1} \wedge \cdots \wedge \omega^{p}, \eta^{1} \wedge \cdots \wedge \eta^{p}\right)=\operatorname{det}\left(g_{x}\left(\omega^{i}, \eta^{j}\right)\right), \quad \omega^{i}, \eta^{j} \in M_{x}^{*}$.

We extend $g$ to the exterior algebra $\Lambda\left(M_{x}^{*}\right)$ by requiring that the inner product of forms of different order vanish.

Communicated by I. M. Singer, February 15, 1980. 
The orientation of $M$ provides us with a distinguished connected component of the punctured line $\Lambda^{m}\left(M_{x}^{*}\right)-0$, and thus an $E_{x} \in \Lambda^{m}\left(M_{x}^{*}\right)$ with $g_{x}\left(E_{x}, E_{x}\right)=(-1)^{q}$. The Hodge operator is the unique linear operator $*$ on $\Lambda\left(M_{x}^{*}\right)$ carrying $\Lambda^{p}\left(M_{x}^{*}\right) \rightarrow \Lambda^{m-p}\left(M_{x}^{*}\right)$ and satisfying

$$
\begin{aligned}
* E_{x} & =(-1)^{q}, \\
g_{x}(\omega, \eta) E_{x} & =*(\omega \wedge * \eta) .
\end{aligned}
$$

The right-hand side of each equation may be viewed as a real number because $\Lambda^{0}\left(M_{x}^{*}\right) \cong \mathbf{R}$ canonically. We also denote by $*$ the induced operator on section spaces of $\Lambda\left(T^{*}(M)\right)$; in particular on smooth differential forms.

Both the Hodge * and the exterior derivative $d$ are "unchanged" in their action on forms which take their "values" in a real vector space $V$; that is, on sections of $V \otimes_{\mathbf{R}} \Lambda\left(T^{*}(M)\right)$. Any choice of a basis $v_{1}, \cdots, v_{n}$ for $V$ allows us to write

$$
\begin{aligned}
*\left(v_{j} \otimes \omega^{j}\right)=v_{j} \otimes * \omega^{j} & \text { (summation convention), } \\
d\left(v_{j} \otimes \omega^{j}\right)=v_{j} \otimes d \omega^{j}, & \omega^{j} \in \Lambda\left(M_{x}^{*}\right),
\end{aligned}
$$

and these formulas are basis-independent.

If $V$ is actually a Lie algebra $g$, we may generalize the wedge product of $\mathbf{R}$-valued forms to the bracket of $\mathrm{g}$-valued forms. In the notation above,

$$
\left[v_{j} \otimes \omega^{j}, v_{k} \otimes \eta^{k}\right]=\left[v_{j}, v_{k}\right] \omega^{j} \wedge \eta^{k}
$$

This product satisfies the $\mathbf{Z}_{2}$-graded anticommutativity law and Jacobi identity:

$$
\begin{gathered}
{[\Xi, \Omega]=(-1)^{p q+1}[\Omega, \Xi],} \\
(-1)^{p r}[\Xi,[\Omega, \Psi]]+(-1)^{q p}[\Omega,[\Psi, \Xi]]+(-1)^{r q}[\Psi,[\Xi, \Omega]]=0, \\
\Xi \in \mathrm{g} \otimes \Lambda^{p}\left(M_{x}^{*}\right), \quad \Omega \in \mathrm{g} \otimes \Lambda^{q}\left(M_{x}^{*}\right), \quad \Psi \in \mathrm{g} \otimes \Lambda^{r}\left(M_{x}^{*}\right) .
\end{gathered}
$$

We may also wedge a real-valued form with a $g$-valued form, this operation being characterized by the formula

$$
\omega \wedge\left(v_{j} \otimes \eta^{j}\right)=v_{j} \otimes\left(\omega \wedge \eta^{j}\right) ; \omega, \eta^{j} \in \Lambda\left(M_{x}^{*}\right),
$$

and satisfying

$$
d(\omega \wedge \Omega)=d \omega \wedge \Omega+(-1)^{p} \omega \wedge d \Omega,
$$

where $\omega$ is a smooth $\mathbf{R}$-valued $p$-form, and $\Omega$ is a smooth $g$-valued form. 
The Yang-Mills equations may be stated as follows. If $A$ is a $g$-valued one-form on $M$, the covariant derivative of a $g$-valued $p$-form $\Omega$ with respect to $A$ is

$$
d_{A} \Omega=d \Omega-e_{p}[A, \Omega],
$$

where $e_{p}$ is a nonzero coupling constant depending on $p$. Choosing $e_{2}=2 e_{1}$ results in the Bianchi identity $d_{A} d_{A} A=0$. Here we assume only $e_{2}=2 e_{1} \equiv e^{\prime}$, and define $e_{m-1} \equiv e$.

The Yang-Mills equations are

$$
F=d_{A} A, \quad d_{A} * F=0 .
$$

The one-form $A$ is called the connection (in geometry) or potential (in physics); $F$ is called the curvature form or field strengths.

\section{Conformal transformations and vector fields}

The following definitions and lemmas are contained in [3].

Definition 3.1. (a) Let $M$ and $N$ be pseudoriemannian manifolds of signature $(k, q)$ equipped with pseudometrics $g_{M}$ and $g_{N}$ respectively. A diffeomorphism $h: M \rightarrow N$ is a conformal transformation if $h^{*} g_{N}=\gamma g_{M}$ for some positive $\gamma \in C^{\infty}(M, \mathbf{R})$, where $h^{*}$ is the pullback of covariant tensors under $h$. A conformal transformation on $M$ is a conformal transformation $M \rightarrow M$.

(b) A smooth vector field $X$ on $M$ is conformal if $\theta(X) g_{M}=\rho g_{M}$ for some $\rho \in C^{\infty}(M, \mathbf{R})$. Here $\theta(X)$, the Lie derivative, is the unique type-preserving derivation on the mixed tensor algebra $\mathscr{D}(M)$ which extends $f \mapsto X f$ on functions and $Y \mapsto[X, Y]$ on vector fields, and which commutes with contractions [2].

(c) A conformal vector field $X$ is locally integrable to a local one-parameter group of conformal transformations if for each $x \in M$ there are an open set $U_{x}$ containing $x$ and a local one-parameter group $h_{t}$ of conformal transformations "on $U_{x}$ " (between open subsets of $U_{x}$, the domain set always containing $x$ ) with generator $X$ in the sense that $X_{x}$ is tangent to $t \mapsto h_{t}(x)$ at $t=0$.

Remark 3.2. (a) The set of conformal transformations on $M$ forms a group under composition.

(b) Let $h$ be a conformal transformation $M \rightarrow N$. Since $h$ is a diffeomorphism, $h^{*}\left(g_{N}\right)_{h(x)}$ is necessarily nondegenerate on $M_{x}$; furthermore, it has signature $(k, q)$, the same as $\left(g_{N}\right)_{h(x)}$. Thus the hypothesis $\gamma>0$ is superfluous unless $m$ is even and $k=q=m / 2$. 
(c) In the situation of part (c) of Definition 3.1, the action of $\theta(X)$ on covariant tensors (real or vector-valued) is given by

$$
(\theta(X) \Omega)_{x}=\left.\frac{d}{d t} h_{t}^{*} \Omega_{h_{t}(x)}\right|_{t=0} .
$$

If $h_{t}^{*} g=\gamma_{t} g$, application of (3.1) with $\Omega=g$ yields $\theta(X) g=\rho g$, where

$$
\rho(x)=\left.\frac{d}{d t} \gamma_{t}(x)\right|_{t=0}
$$

(d) In most applications, the manifolds $M$ and $N$ are open subsets of such manifolds as Minkowski space or its conformal compactification [5].

The properties which are crucial to the quasi-invariance relations for the Yang-Mills equations describe the behavior of the Hodge * relative to conformal transformations and vector fields. We let $\mathscr{D}_{p}(M, \mathfrak{g})$ denote the space of smooth $g$-valued $p$-forms on $\boldsymbol{M}$.

Lemma 3.3. (a) If $h$ is a conformal transformation $M \rightarrow N, h^{*}\left(g_{N}\right)=\gamma g_{M}$, then

$$
* h^{*} \Omega= \pm \gamma^{-(m-2 p) / 2} h^{*}(* \Omega), \quad \Omega \in \mathscr{D}_{p}(N, \mathfrak{g}),
$$

the plus sign taken if $h$ is orientation-preserving $\left(h^{*} E_{N}=\delta E_{M}, \delta \in\right.$ $C^{\infty}(M, \mathbf{R})$ with $\left.\delta>0\right)$, and the minus if $h$ is orientation-reversing $(\delta<0)$.

(b) If $X$ is a conformal vector field on $M, \theta(X) g_{M}=\rho g_{M}$, which is locally integrable to a local one-parameter group of conformal transformations, then

$$
* \theta(X) \Omega=\theta(X) * \Omega-\frac{1}{2}(m-2 p) \rho * \Omega, \quad \Omega \in \mathscr{D}_{p}(M, g) .
$$

Proof. (a) It is clearly enough to prove (3.3) with a real-valued $p$-form $\omega$ in place of $\Omega$.

If $\varphi$ is a real-valued one-form on $N$, the identification of tangent and cotangent spaces given by $g_{M}$ identifies $h^{*} \varphi$ with $\gamma\left(d h^{-1}\right) X_{\varphi}$, where $X_{\varphi}$ is identified with $\varphi$ through $g_{N}$. Thus

$$
\begin{aligned}
g_{M}\left(h^{*} \varphi, h^{*} \psi\right) & =\gamma^{2} g_{M}\left(\left(d h^{-1}\right) X_{\varphi},\left(d h^{-1}\right) X_{\psi}\right) \\
& =\gamma\left(h^{*} g_{N}\right)\left(\left(d h^{-1}\right) X_{\varphi},\left(d h^{-1}\right) X_{\psi}\right) \\
& =\gamma g_{N}\left(X_{\varphi}, X_{\psi}\right) \circ h \\
& =\gamma g_{N}(\varphi, \psi) \circ h,
\end{aligned}
$$

where $\varphi, \psi \in \mathscr{D}_{1}(N, \mathbf{R})$. Now if $\omega, \eta \in \mathscr{D}_{p}(N, \mathbf{R})$, then (2.1) gives

$$
g_{M}\left(h^{*} \omega, h^{*} \eta\right)=\gamma^{p} g_{N}(\omega, \eta) \circ h .
$$

In particular,

$$
g_{M}\left(h^{*} E_{N}, h^{*} E_{N}\right)=\gamma^{m}(-1)^{q}
$$


so that $h^{*} E_{N}= \pm \gamma^{m / 2} E_{M}$. Thus taking $h^{*}$ of both sides of (2.3) in the form

$$
g_{N}(\omega, \eta) E_{N}=\eta \wedge * \omega
$$

yields

$$
\begin{aligned}
& \pm \gamma^{(m-2) / 2} h^{*} \eta \wedge * h^{*} \omega \\
& \quad=\left[\gamma^{-p} g_{M}\left(h^{*} \omega, h^{*} \eta\right)\right]\left( \pm \gamma^{m / 2} E_{M}\right) \\
& \quad=h^{*} \eta \wedge h^{*}(* \omega) .
\end{aligned}
$$

Because an $(m-p)$-form on $M$ is determined by its wedge products with elements of $\mathscr{D}_{p}(M, \mathbf{R})$ and thus by its wedge with the $h^{*} \eta,(3.3)$ follows.

(b) Let $h_{t}$ be the local one-parameter group of conformal transformations generated by $X$, so that $h_{t}^{*} g_{M}=\gamma_{t} g_{M}$. Since $h_{0}$ is the identity, continuity implies that all $h_{t}$ preserve orientation. If $\Omega \in \mathscr{D}_{p}(M, \mathfrak{g})$, then (3.1), (3.2), and (3.3) give

$$
\begin{aligned}
(\theta(X) * \Omega)_{x} & =\left.\frac{d}{d t} h_{t}^{*}(* \Omega)_{h_{t}(x)}\right|_{t=0} \\
& =\left.\frac{d}{d t}\left(\gamma_{t}(x)^{(m-2 p) / 2} * h_{t}^{*} \Omega_{h_{t}(x)}\right)\right|_{t=0} \\
& =*\left(\left.\frac{d}{d t} h_{t}^{*} \Omega_{h_{t}(x)}\right|_{t=0}+\frac{1}{2}(m-2 p)\left(\left.\frac{d}{d t} \gamma_{t}(x)\right|_{t=0}\right) \Omega_{x}\right) \\
& =*\left((\theta(X) \Omega)_{x}+\frac{1}{2}(m-2 p) \rho(x) \Omega_{x}\right),
\end{aligned}
$$

which is equivalent to (3.4).

We note finally that the relations

$$
\begin{aligned}
h^{*}(\omega \wedge \eta) & =h^{*} \omega \wedge h^{*} \eta, \\
\theta(X)(\omega \wedge \eta) & =\omega \wedge \theta(X) \eta+\theta(X) \omega \wedge \eta
\end{aligned}
$$

for real-valued differential forms imply the relations

$$
\begin{gathered}
h^{*}[\Xi, \Omega]=\left[h^{*} \Xi, h^{*} \Omega\right], \\
\theta(X)[\Xi, \Omega]=[\Xi, \theta(X) \Omega]+[\theta(X) \Xi, \Omega]
\end{gathered}
$$

for $\mathfrak{g}$-valued forms.

\section{Quasi-invariance of the Yang-Mills equations}

For a nonlinear differential equation, three types of quasi-invariance relations are relevant:

(1) quasi-invariance of the equations under conformal transformations;

(2) quasi-invariance of the linearized equations under conformal transformations; 
(3) quasi-invariance of the linearized equations under Lie derivation with respect to conformal vector fields.

We set $y(A)=d_{A} * d_{A} A$ for $A \in \mathscr{D}_{1}(M, \mathfrak{g})$; that is, $y$ is the nonlinear function on $\mathscr{D}_{1}(M, g)$ whose zeros are solutions of the Yang-Mills equations. As for the linearized equations, we make the following definition.

Definition 4.1. Let $V$ and $W$ be real vector spaces, and let

$$
M_{j}: V \times \underset{j \text { times }}{\ldots} \times V \rightarrow W
$$

be a $j$-linear function for $0 \leqslant j \leqslant N$. The linearization of the equation

$$
\sum_{j=0}^{N} M_{j}(v, \cdots, v)=0
$$

at $v \in V$ is the equation

$$
\sum_{j=0}^{N}\left[\sum_{i=1}^{j} M_{j}(v, \cdots, \underset{\substack{\uparrow \\ i \text {-th place }}}{X, \cdots, v)})=0\right.
$$

as a condition on $X \in V$.

Thus the linearization of the Yang-Mills system

$$
\begin{aligned}
& F=d_{A} A=d A-\frac{e^{\prime}}{2}[A, A], \\
& 0=d_{A} * F=d * F-e[A, * F],
\end{aligned}
$$

at $A \in \mathscr{D}_{1}(M, g)$ is

$$
\begin{aligned}
f & =d a-e^{\prime}[A, a] \quad(\text { by }(2.5)), \\
0 & =d * f-e[a, * F]-e[A, * f] \\
& =d_{A} * f-e[a, * F], \quad F=d_{A} A,
\end{aligned}
$$

as a condition on $a \in \mathscr{D}_{1}(M, g)$. We define the linear function $Y_{A}: \mathscr{D}_{1}(M, g)$ $\rightarrow \mathscr{Q}_{m-1}(M, g)$ by

$$
\begin{aligned}
Y_{A} a & =d_{A} * f-e[a, * F], \\
f & =d a-e^{\prime}[A, a], \quad F=d_{A} A .
\end{aligned}
$$

Theorem 4.2. Let $A \in \mathscr{D}_{1}(M, \mathrm{~g})$ and $F=d_{A} A$.

(a) If $h$ is a conformal transformation $M \rightarrow N, h^{*} g_{N}=\gamma g_{M}$, then

$$
\begin{aligned}
y\left(h^{*} A\right) & = \pm\left(\gamma^{(4-m) / 2} h^{*} y(A)-\frac{1}{2}(m-4) \gamma^{(2-m) / 2} d \gamma \wedge h^{*}(* F)\right) \\
Y_{h^{*} A} h^{*} a & = \pm\left(\gamma^{(4-m) / 2} h^{*} Y_{A} a-\frac{1}{2}(m-4) \gamma^{(2-m) / 2} d \gamma \wedge h^{*}(* f)\right) \\
f & =d a-e^{\prime}[A, a] .
\end{aligned}
$$


As usual, we take the plus sign if h preserves orientation, and the minus sign if $h$ reverses orientation.

(b) If $X$ is a conformal vector field on $M, \theta(X) g_{M}=\rho g_{M}$, which is locally integrable to a local one-parameter group of conformal transformations which fix $A$, then

$$
\begin{gathered}
Y_{A} \theta(X) a=\theta(X) Y_{A} a-\frac{1}{2}(m-4)\left\{d_{A}(\rho * f)-e \rho[a, * F]\right\}, \\
f=d a-e^{\prime}[A, a] .
\end{gathered}
$$

Proof. (a) We calculate

$$
\begin{gathered}
y\left(h^{*} A\right)=d_{h^{*} A} * F^{\prime}, \\
F^{\prime}=d_{h^{*} A} h^{*} A=d h^{*} A-\frac{e^{\prime}}{2}\left[h^{*} A, h^{*} A\right]=h^{*} F .
\end{gathered}
$$

By (3.3),

$$
\begin{aligned}
y\left(h^{*} A\right) & =d_{h^{*} A}\left( \pm \gamma^{(4-m) / 2} h^{*}(* F)\right) \\
& = \pm\left(d\left(\gamma^{(4-m) / 2} h^{*}(* F)\right)-e \gamma^{(4-m) / 2}\left[h^{*} A, h^{*}(* F)\right]\right) \\
& = \pm\left(\gamma^{(4-m) / 2} h^{*} d_{A} * F-\frac{1}{2}(m-4) \gamma^{(2-m) / 2} d \gamma \wedge h^{*}(* F)\right) \\
& = \pm\left(\gamma^{(4-m) / 2} h^{*} y(A)-\frac{1}{2}(m-4) \gamma^{(2-m) / 2} d \gamma \wedge h^{*}(* F)\right)
\end{aligned}
$$

To prove (4.2), set $f=d a-e^{\prime}[A, a]$, and calculate

$$
\begin{aligned}
Y_{h^{*} A} h^{*} a & =d_{h^{*} A} * f^{\prime}-e\left[h^{*} a, * h^{*} F\right] \\
f^{\prime} & =d h^{*} a-e^{\prime}\left[h^{*} A, h^{*} a\right]=h^{*} f
\end{aligned}
$$

By (3.3),

$$
\begin{aligned}
Y_{h^{*} A} h^{*} a & = \pm\left(d_{h^{*} A}\left(\gamma^{(4-m) / 2} h^{*}(* f)\right)-e \gamma^{(4-m) / 2} h^{*}[a, * F]\right) \\
= & \pm\left(\gamma^{(4-m) / 2} h^{*} d_{A} * f-\frac{1}{2}(m-4) \gamma^{(2-m) / 2} d \gamma \wedge h^{*}(* f)\right. \\
\left.-e \gamma^{(4-m) / 2} h^{*}[a, * F]\right) & \\
= & \pm\left(\gamma^{(4-m) / 2} h^{*} Y_{A} a-\frac{1}{2}(m-4) \gamma^{(2-m) / 2} d \gamma \wedge h^{*}(* f)\right)
\end{aligned}
$$

(b) Let $h_{t}$ be the one-parameter group generated by $X$, so that $h_{t}^{*} g_{M}=$ $\gamma_{t} g_{M}$. Since the $h_{t}$ fix $A$, (3.1) implies that $\theta(X) A=0$, and the field strength perturbation $f^{\prime}$ associated to $\theta(X) a$ is

$$
f^{\prime}=d \theta(X) a-e^{\prime}[A, \theta(X) a]=\theta(X) f
$$


by (3.6) and the fact that $d$ commutes with $\theta(X)$. Thus

$$
\begin{aligned}
Y_{A} \theta(X) a= & d_{A} * \theta(X) f-e[\theta(X) a, * F] \\
= & d_{A}\left(\theta(X) * f-\frac{1}{2}(m-4) \rho * f\right)-e[\theta(X) a, * F] \\
= & d \theta(X) * f-e[A, \theta(X) * f]-\frac{1}{2}(m-4) d_{A}(\rho * f) \\
& -e[\theta(X) a, * F] \\
= & \theta(X) d_{A} * f-\frac{1}{2}(m-4) d_{A}(\rho * f)-e[\theta(X) a, * F] \\
= & \theta(X) d_{A} * f+\frac{1}{2}(m-4) d_{A}(\rho * f)-e \theta(X)[a, * F] \\
& +e[a, \theta(X) * F] .
\end{aligned}
$$

Now $\theta(X) * F=* \theta(X) F+\frac{1}{2}(m-4) \rho * F$, which simplifies to $\frac{1}{2}(m-4) \rho$ $* F$ as $\theta(X) F=\theta(X)\left(d A-\frac{1}{2} e^{\prime}[A, A]\right)=d \theta(X) A-e^{\prime}[A, \theta(X) A]=0$. This makes the above

$$
\theta(X) Y_{A} a-\frac{1}{2}(m-4)\left\{d_{A}(\rho * f)-e \rho[a, * F]\right\} .
$$

Remark 4.3. (a) The Theorem points up the importance of dimension 4 in the Yang Mills theory as $m=4$ reduces (4.1)-(4.3) to

$$
\begin{aligned}
y\left(h^{*} A\right) & =h^{*} y(A), \\
Y_{h^{*} A} h^{*} a & =h^{*} Y_{A} a, \\
Y_{A} \theta(X) a & =\theta(X) Y_{A} a .
\end{aligned}
$$

The signature $(k, q)$ of the pseudometric is irrelevant to these formulas; in particular, it may be $(4,0)$ as in the case of Euclidean Yang-Mills (studied by Atiyah, Singer, et al), or $(3,1)$ as in the case of the equations in their original physical (hyperbolic) form, as studied by Segal.

(b) In any dimension, the Yang-Mills equations and their linearizations are invariant under uniform dilations $\left(h^{*} g_{N}=a g_{M}, a>0\right.$ constant), and in particular, under isometries $(a=1)$, since for such $h, d \gamma=0$ in (4.1) and (4.2). For isometries, we again have (4.4) and (4.5). If a conformal vector field $X$ integrates to a local one-parameter group of uniform dilations, the $\rho$ in $\theta(X) g_{M}=\rho g_{M}$ is constant by (3.1), so that (4.3) becomes

$$
Y_{A} \theta(X) a=\left\{\theta(X)-\frac{1}{2}(m-4) \rho\right\} Y_{A} a,
$$

and we have invariance. If $X$ integrates to a local one-parameter group of isometries, $\theta(X) g_{M}=0$ and we again have (4.6).

(c) For (4.2) and (4.3), it was not necessary to assume that the "background" potential $A$ satisfy the Yang-Mills equations. 


\section{References}

[1] T. P. Branson, The Yang-Mills equations: quasi-invariance, special solutions, and Banach manifold geometry, Ph.D. dissertation, Massachusetts Institute of Technology, Cambridge, 1979.

[2] S. Helgason, Differential geometry and symmetric spaces, Academic Press, New York, 1962.

[3] B. Ørsted, Wave equations, particles, and chronometric geometry, Ph.D. dissertation, Massachusetts Institute of Technology, Cambridge, 1976.

[4] A note on the conformal quasi-invariance of the Laplacian on a pseudoriemannian manifold, Lett. Math. Phys. 1 (1977) 183.

[5] I. E. Segal, Mathematical cosmology and extragalactic astronomy, Academic Press, New York, 1976.

[6] General properties of the Yang-Mills equations in physical space, Proc. Nat. Acad. Sci. U.S.A. 75 (1978) 4638-4639.

[7] The Cauchy problem for the Yang-Mills equations, J. Functional Analysis 33 (1979) 175-194.

PURDUE UNIVERSITY 
